\title{
$\infty$ \\ INTELIGÊNCIA ArTIFICIAL E A TRANSFORMAÇÃO DIGITAL EM MEDICINA
}

\section{Roberto Vieira Botelho ${ }^{a}$}

${ }^{\text {a }}$ Presidente da Fundação Adib Jatene.

E-mail: robertobotelho@mac.com

\section{Resumo}

A possibilidade de codificação digital da informação tem transformado a vida das pessoas em velocidade exponencial. Três variáveis se destacam para essa transformação digital: a geração de grandes arquivos digitais, superiores às capacidades de processamento dos atuais computadores (Bigdata); a alta velocidade e disponibilidade universal da Internet; e o compartilhamento desses arquivos em nuvem, possibilitando o compartilhamento de super-máquinas. Máquinas com alta capacidade de processamento por segundo passam a executar funçóes, que, se realizadas por humanos, exigiriam inteligência. É a inteligência artificial. As atividades que exibem padrão repetitivo passam, progressivamente, a ser melhor executadas por computadores substituindo ou apoiando e melhorando as tarefas humanas. As grandes vias de telecomunicação ampliam a trans- formação através do exercício da medicina à distância: a telemedicina. $\mathrm{O}$ valor dos dados supera o valor dos serviços de saúde. Pacientes, profissionais e organizaçôes de saúde são demandadas a adaptaçôes rápidas e redefiniçôes de seus papeis, destacando-se o foco no paciente, que assume seu protagonismo. Esse ambiente gera previsóes otimistas com impactos significativos sobre indicadores relevantes de saúde. Mas também, são acompanhados por possibilidades preocupantes, especialmente as relacionadas à segurança $e$ privacidade das pessoas ou mesmo de países, sob o conceito da ditadura digital.

\section{Palavras Chave}

Inteligência artificial, telemedicina, saúde digital

\section{Abstract}

The possibility of coding information has been changing people's lives at exponential speeds. Three variables have stood out in this digital transformation: the production of large digital files, demanding 
a higher processing capacity than that of our current computers (Big Data); the high speed and universal availability of the Internet; and the cloud-based file sharing, which enables sharing super machines. Machines with a high processing capacity per second have started executing functions that would require the use of intelligence if carried out by humans. It is the artificial intelligence. Activities with a repetitive pattern have progressively been better executed by computers, which have replaced or supported and enhanced human tasks. The large telecommunication networks amplify this transformation via distance medicine: the telemedicine. The value of data is higher than that of health care services. Patients, health professionals, and health organizations have had to quickly adapt themselves and redefine their roles, particularly concerning patient-centered care, which has become a protagonist. This scenario presents an optimistic outlook with significant impact on relevant health indicators. However, it comes with worrying possibilities, particularly those regarding safety and privacy of people or even countries, in light of the concept of digital dictatorship.

\section{Keywords}

Artificial intelligence, telemedicine, digital health.

\section{INTRODUÇÁO}

O tempo entre o desenvolvimento de um produto e sua aplicação no mundo real vem se encurtando progressivamente, fenômeno conhecido como transformação histórica. A comparação entre o tempo de desenvolvimento da fotografia, do rádio, da televisão até a evolução desde os computadores 486, até o Pentium, demonstram essas diferenças. Essa curva, é exponencial a partir do desenvolvimento dos computadores ${ }^{1}$. Desde 1965, o número de transistores por Chip dobra a cada 18 meses, o que é conhecido como a lei de Moore. A partir de 2010, essa capacidade tem se limitado, quando aparece a alternativa da computaçáo quântica $^{2}$. Em Outubro de 2019, pesquisadores da Google e NASA publicaram a chamada " supremacia Quântica” ao realizarem em 200 milissegundos o que os melhores computadores atuais fariam em $10 \mathrm{mil} \mathrm{anos}^{3}$.

Outro fenômeno transformador de nossa realidade tem sido a capacidade das telecomunicaçóes. Em 1997, para se baixar um vídeo de alta resolução de de 800 Megabytes, consumiam-se 36 horas através de conexôes GPRS (50 kilobits/s). Hoje, através de conexão 5G (6400 megabits/s), o mesmo vídeo é baixado em 1 segundo ${ }^{4}$. Soma-se a essa velocidade de comunicação, o progressivo acesso de massas populacionais à Internet.

Cerca de $90 \%$ dos norte americanos, $87 \%$ dos europeus, $68 \%$ dos latino ameri- 
canos e $51 \%$ da população mundial, estão conectados à Internet ${ }^{5}$. Como consequência, o volume de dados trafegados e hospedados a baixo custo cria o "big data". Observa-se crescimento de $40 \%$ por ano do volume de geração de dados, contra um aumento de $5 \%$ nos gastos de tecnologia da informação. A biblioteca dos congresso dos Estados Unidos da América armazenou, até abril de 2011, 235 terabytes de dados. Os dados acumulados por empresa foi maior que isso em $15 \mathrm{de}$ 17 setores da economia americana. Quando o volume desse conjunto de dados é maior que a capacidade das ferramentas de software para capturar, armazenar, processar e analisar, define-se o " bigdata”. Essa definição é subjetiva e dinâmica, pois varia conforme a evoluçáo da tecnologia. À medida que esta evolui, um certo volume de dados deixa de ser bigdata. $\mathrm{O}$ valor potencial atribuído ao bigdata em saúde nos EUA é de cerca de 300 bilhões de dólares por ano, mais que o dobro do gasto em saúde da Espanha. A necessidade de novos gestores de dados para conduzirem o bigdata em saúde americano é estimada em 1,5 milhôes de novos profissionais ${ }^{6}$. Contemporaneamente, surge a computação nas nuvens e consequente compartilhamento de capacidade de processamento computacional. Esse é o substrato da transformação

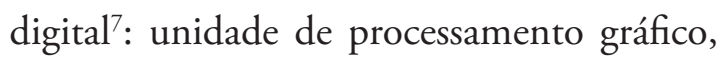
big data e computação nas nuvens (Fig 1.).

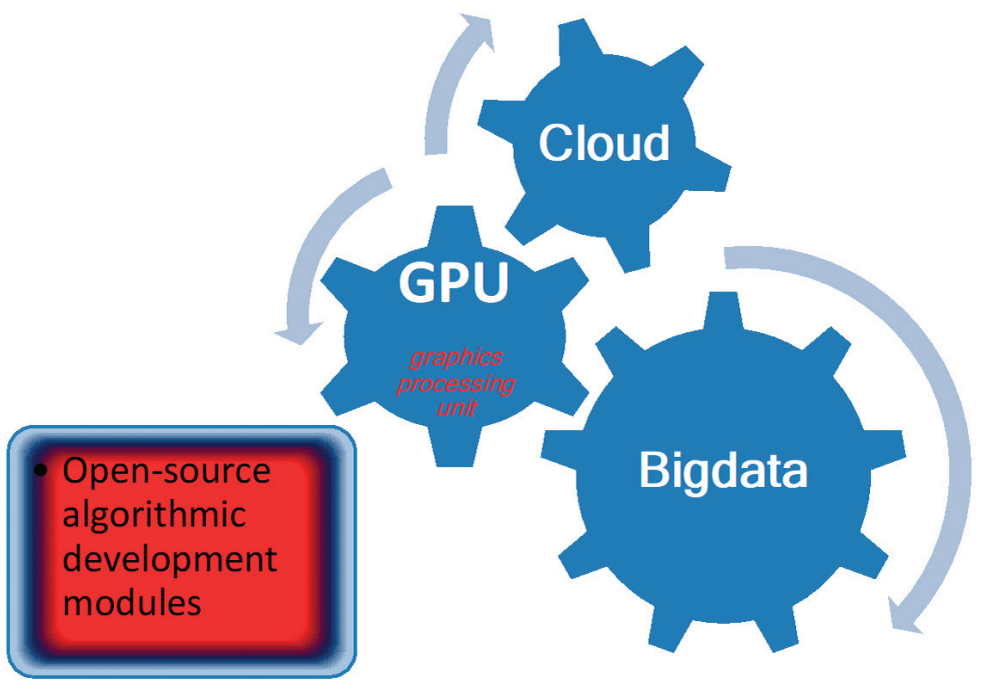

Fig 1. Na medicina, observa-se progressiva digitalização das informaçóes e seu arquivo estruturado em plataformas dedicadas a imagens (Picture archiving and communications systems- PACS), a laboratórios (Laboratory Information Systems-LIS) e à gestão hospitalar (Hospital Information Systems- HIS). Com isso, o profissional de saúde se vê pressionado por sobrecarga de informaçôes, cobrança de eficiência operacional e medicina personalizada. 
Associe-se a isso, o custo crescente da implementação tecnológica em saúde. $\mathrm{O}$ usuário do sistema de saúde deixa de ser "paciente" e se transforma em consumidor apoderado. Uma pessoa que utilize relógios com sensores, conectados a telefones inteligentes e à internet, pode receber alertas sobre indicadores de saúde e procurar assistência médica. Este "usuário" chega ao consultório com informaçôes sobre seu risco ou doença. Já sabe o prognóstico, qual o melhor tratamento, qual farmácia obtém medicamentos sob melhor custo e quais as publicaçóes do médico.

\section{A InTeligênCIA ArTificial}

Desde 1936 registram-se publicaçóes sobre emprego de algoritmos matemáticos para resolução de problemas, especialmente aqueles relacionados a padróes repetitivos ${ }^{8}$. A disponibilidade de grandes volumes de dados, provenientes de sensores, dispositivos de fitness, equipamentos biomédicos, telefones inteligentes, clima, poluição, qualidade do ar, informaçóes experimentais de laboratórios, genômica, proteômica, microbioma, informações clínicas estruturadas ou não, a serem processados por potentes computadores compartilhados passa a gerar modelos computacionais a partir de algoritmos. Os computadores são alimentados de maneira padronizada (regressão logística, árvore de decisão, redes neurais, aprendizado de máquina) supervisionados ou não e geram modelos para validação clinica ${ }^{9}$. Esses algoritmos podem contribuir com três grandes áreas da saúde: a pesquisa e desenvolvimento, a assistência e a gestão. Quanto à pesquisa e desenvolvimento, observa-se aplicação imediata, intensamente estimulada pelo ecossistema, seja para integraçáo de dados de genômica, descoberta de novos agentes terapêuticos, especialmente alvos precisos ou pela estratificação de doenças. $\mathrm{O}$ mesmo se observa quanto à gestáo, destacando-se a otimizaçáo da alocaçáo de recursos, robótica, monitoramento continuo. Já na assistência, o emprego de inteligência artificial convive com dificuldades, tanto imanentes à ampla variabilidade do fenômeno biológico, quanto à resistência dos profissionais em implementar algoritmos, além da dificuldade regulatória: quem certifica? Como avaliar um algoritmo que deu errado, se não se conhece de onde ele coletou variáveis com padrão repetitivo? Ademais, há grande preocupaçáo da substituição de tarefas repetitivas, que passam a ser melhor desempenhadas por máquinas. Outro elemento paradigmático nesta evolução da inteligência artificial é o conceito de pensamento antropomórfico reverso, em que o homem é condicionado a exigir que o processamento da máquina siga sua lógica de pensamento humano. Na maioria das vezes, o criador náo sabe que variáveis o algoritmo utiliza para entregar seus resul$\operatorname{tados}^{10}$. Os algoritmos são uma "caixa preta". 
Um exemplo desse fenômeno foi observado quando propusemos a detecção do infarto do miocárdio a partir de uma só derivação de eletrocardiograma ${ }^{11}$. O algoritmo identifica padrôes repetitivos distintos do processamento gráfico mental humano. Assim, a derivação D1 do eletrocardiograma, registrada através de um relógio de pulso, onde um eletrodo está embutido na caixa do relógio e o outro, na coroa, consegue diagnosticar infarto de quaisquer segmentos miocárdicos.

\section{O PROBLEMA DA SEGURANÇA E PRIVACIDADE}

A velocidade das publicaçôes sobre inteligência Artificial traz previsóes preocupantes. O seu emprego pode se dar para o bem, ou não. Alguns autores alertam para o perigo da ditadura digital. A liderança em desenvolvimentos de algoritmos, por grupos econômicos, ou países, pode criar hiatos abissais entre os povos. Previsóes pessimistas apontam para o desaparecimento de milhóes de empregos. Apesar do aparecimento de outros milhóes, estratos mais carentes das populaçóes mundiais, ou mesmo países inteiros, terão menos capacidade de re-treinamento e realocaçáo a esse mercado de trabalho volátil, gerando massas de "inúteis", como sugere Yuval Hara$\mathrm{ri}^{12}$. Algumas especialidades cuja prática predominante seja o processamento de dados, sob padrão repetitivo, como a radiologia, dermatologia, algumas áreas da oftalmologia, pediatria, entre outras, sofrerão grande impacto, pois os algoritmos serão, alguns já são, melhores e mais custo/efetivos que os humanos. $\mathrm{O}$ modelo de prestação de serviços em saúde migra para organizaçôes "accountable", não há tradução adequada para o Português. Assim, as organizações que implementarem modelos de inteligência artificial na assistência e na gestão, terão significativos ganhos em eficiência e entregarão muito mais valor aos usuários.

À medida que os bancos de dados ganhem valor, e ganharão, aumentará o interesses de grupos mal intencionados. Já se observam sequestros de bancos de dados de hospitais e clinicas. Alguns algoritmos tem sido desenvolvidos para combater outros, gerando uma batalha digital (algoritmo $\mathrm{x}$ algoritmo).

\section{Conclusão}

A transformação digital nos encaminha para a singularidade, sugerida por Raymond Kurzweil, momento em que a maioria da informação disponível para a humanidade esteja sobre forma de energia. Neste momento, haverá importante convergência biológicodigital e o homem não se ocupará de atividades triviais, deixando-as aos robôs. Esse ambiente gera ciclo virtuoso, com aumento da renda per-capita e da expectativa de vida com qualidade. 


\section{REFERÊNCIAS BIBLIOGRÁFICAS}

1. Kurzweil, Ray. The Singularity Is Near: When Humans Transcend Biology. New York : Penguin, 2006. Print.

2. John Loeffler. Available at https://interestingengineering.com/no-more-transistors-the-end-of-moores-law Accessed on $11 / 6 / 2019$.

3. Arute, F., Arya, K., Babbush, R. et al. Quantum supremacy using a programmable superconducting processor. Nature 574, 505-510 (2019) doi:10.1038/s41586-019-1666-5

4. Chris Hoffman. How fast will $5 \mathrm{G}$ be? Available at: https://www.howtogeek. com/340002/what-is-5g-and-how-fastwill-it-be/ Accessed on 11/6/2019.

5. Internet users in the world. Available at: https://www.internetworldstats.com/ stats.htm Accessed on 11/6/2019

6. James Manyika et al. Big data: The next frontier for innovation, competition, and productivity. McKinsey Global Institute. May 2019.
7. Beam AL, Kohane IS. Translating Artificial Intelligence into Clinical Care. JAMA December 13, 2016 Volume 316, Number 22.

8. Turing, A. M., "On Computable Numbers with an Application to the Entscheidungsproblem." Proceedings of the London Mathematical Society, 1936.42(1):pp.230-265.doi: 10.1112/ $\mathrm{plms} / \mathrm{s} 2-42.1 .230$.

9. Johnson K.W. et al. The Role of AI in Medicine. J Am Coll Cardiol. 2018;71(23):2668-79.

10. Eric Topol. Deep Medicine. How Artificial Intelligence Can Make Healthcare Human Again. Hachette UK. Basic Books, 2019. Print.

11. Metha, $S$ et al. Application of Artificial Intelligence to Detect ST Elevation MI with a Single Lead EKG. Journal of the American College of Cardiology 73(9):1328 DOI: 10.1016/S07351097(19)31935-7

12. Yuval Harari. 21 Lessons for the 21 st Centure. Spiegel \&amp; Grau 2018. 